\title{
Response to Letter from Reddy et al.
}

\author{
Anne K. Jensen, MD ${ }^{1}$ and Gil Binenbaum, MD, MSCE ${ }^{1,2}$ \\ ${ }^{1}$ Division of Ophthalmology, The Children's Hospital of Philadelphia, Philadelphia PA \\ 2Department of Ophthalmology, Scheie Eye Institute, Raymond and Ruth Perelman School of \\ Medicine at the University of Pennsylvania, Philadelphia PA
}

The authors respond:

We are grateful to Doctors Reddy, Patel, and Sinha for their kind comments and interest. ${ }^{1}$ It is interesting that their UK study ${ }^{2}$ and our Philadelphia study ${ }^{3}$ demonstrated similar IGF-1 levels in premature infants, but these levels were different in magnitude from the Swedish cohort. ${ }^{4}$ Perhaps this observation is due to the IGF-1 assay used in each study. In both the UK and Philadelphia studies, an immunoenzymatic assay was used, ${ }^{2,3}$ while in the Swedish study, a radioimmunoassay was used. ${ }^{4}$

With regards to the modifying effect of race on the association between low serum IGF-1 and ROP, the UK study and Philadelphia study differed, as the former found an effect of race, ${ }^{2}$ and the Philadelphia study did not. ${ }^{3}$ One explanation is that the racial categories used by investigators may be inconsistent and result in heterogeneous and overlapping groups. This may explain inconsistencies across studies with regards to the association between race and ROP in the past. Doctors Reddy, Patel, and Sinha pose questions, which we answer below.

We chose postmenstrual (PMA) weeks 28 to 33 by beginning with weeks 30 to 33 and then in post-hoc analysis performing a weekly comparison between ROP groups, with regards to IGF-1 levels. The association appeared to be present for weeks 28 and 29 as well, so we expanded the window. We did not examine growth in our analysis. However, we recently reported the design of the Postnatal Growth and ROP (G-ROP) Study, ${ }^{5}$ which enrolled nearly 7,500 infants in the United States and Canada, and collected detailed growth and ROP data. We will investigate the effect the race on risk of ROP using those data. Two infants in our cohort received treatment for ROP. Both infants were identified as Black/African American. They were in fact treated at later ages, PMA weeks 47 and 41, but the former first developed stage 3 disease at PMA week 37. We agree that a rise in IGF-1 levels is required to trigger the proliferative phase and may explain the higher IGF-1 levels at later PMA in our cohort. Finally, we agree that using IGF-1 levels alone to stratify infants by risk of ROP is impractical due to the small absolute differences between the groups. Postnatal weight gain is a more useful measure.

Corresponding author: Gil Binenbaum MD MSCE, The Children's Hospital of Philadelphia, 3401 Civic Center Blvd, 9-MAIN, Ophthalmology, Philadelphia PA 19104, binenbaum@email.chop.edu, Fax 267-426-5015, Phone 215-590-4594. 


\section{Financial support:}

Our original paper was supported by the National Institutes of Health Grant Numbers NIH K12 EY015398, L30 EY018451-03, 2-P30-EY01583-26, UL1-RR-024134, and 5T35DK060441-10. The content is solely the responsibility of the authors and does not necessarily represent the official views of the National Center for Research Resources or the National Institutes of Health.

\section{References}

1. Reddy MA, Patel HI, Sinha A. Letter to the Editor Regarding Jensen et al's Paper. Retina. 2017, in review.

2. Reddy MA, Patel HI, Karim SM, et al. Reduced Utility of Serum IGF-1 Levels in Predicting Retinopathy of Prematurity Reflects Maternal Ethnicity. The British Journal of Ophthalmology. 2016;100(4):501-504. [PubMed: 26305002]

3. Jensen AK, Ying GS, Huang J, et al. Postnatal Serum Insulin-Like Growth Factor I and Retinopathy of Prematurity. Retina. 2016:0;1-6.

4. Hellstrom A, Engstrom E, Hard AL, et al. Postnatal Serum Insulin-like Growth Factor I Deficiency is Associated with Retinopathy of Prematurity and other Complications of Premature Birth. Pediatrics. 20013;112(5):1016-1020.

5. Binenbaum G, Tomlinson LA. Postnatal Growth and Retinopathy of Prematurity Study: Rationale, Design, and subject Characteristics. Ophthalmic Epidemiol. 2017 2;24(1):36-47. [PubMed: 27996334] 